\title{
Effect of Dephasing on Electron Transport in a Molecular Wire: Green's Function Approach
}

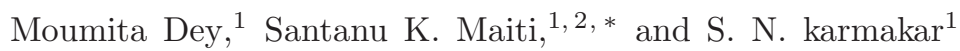 \\ ${ }^{1}$ Theoretical Condensed Matter Physics Division, Saha Institute of Nuclear Physics, \\ Sector-I, Block-AF, Bidhannagar, Kolkata-700 064, India \\ ${ }^{2}$ Department of Physics, Narasinha Dutt College, 129 Belilious Road, Howrah-711 101, India
}

\begin{abstract}
The effect of dephasing on electron transport through a benzene molecule is carefully examined using a phenomenological model introduced by Büttiker. Within a tight-binding framework all the calculations are performed based on the Green's function formalism. We investigate the influence of dephasing on transmission probability and current-voltage characteristics for three different configurations (ortho, meta and para) of the molecular system depending on the locations of two contacting leads. The presence of dephasing provides a significant change in the spectral properties of the molecule and exhibits several interesting patterns that have so far remain unexplored.
\end{abstract}

PACS numbers: 73.63.-b, 73.63.Rt, 85.65.+h, 81.07.Nb

\section{INTRODUCTION}

Ongoing trend of miniaturizing electronic devices eventually approaches the ultimate limit where even a single molecule can be used as an electrical circuit element. Idea of devicing a single molecule as the building block of future generation electronics seems fascinating because of the possibility to assemble a large number of molecules onto a chip i.e., remarkable enhancement in integration density can take place [1]. Discovery of sophisticated molecular scale measurement methodologies such as scanning tunneling microscopy (STM), atomic force microscopy (AFM), scanning electro-chemical microscopy (SECM), etc. have made it possible to study electron transport phenomena in molecular bridge systems [2]. Unlike conventional semiconductor electronic transport properties can not be investigated with Boltzmann transport equation as quantum coherence has a crucial significance on electron transport at this length scale and a full quantum mechanical treatment is needed [3, 4]. At low temperature and small bias electron transport is quite successfully explained within the framework of Landauer formalism [5]. But, validity of Landauer formulation is limited within coherent transport regime where it is assumed that electrons suffer only elastic scattering within the conductor. This assumption allows us to neglect any kind of inelastic processes, which is rather unrealistic unless the time scale related to transport phenomena is much faster than the nuclear motion [6].

A main source of dephasing is electron-phonon (e-ph) interaction in molecular transport junctions. Experimentally the strength of e-ph interaction in molecular systems can be quantified through the measurement of molecular vibrational spectrum using inelastic tunneling spectroscopy [7 -9] . Roughly, it corresponds to finding locations of the peaks in the second order derivative

*Electronic address: santanu.maiti@saha.ac.in of a current-voltage characteristic, where the voltages at the peaks match with eigenenergies of the phonons. Rigorous analysis of e-ph interaction can be done selfconsistently with the help of density functional theory (DFT) and non-equilibrium Green's function formalism (NEGF) [10, 11]. But this method is notably time consuming and becomes very difficult to do even for a molecular conductor comprising a moderate number of atoms. Another way out was proposed by Büttiker over two decades ago [12, 13]. He came up with an elegant idea of incorporating the effect of phase breaking due to inelastic scattering phenomenologically by introducing some fictitious voltage probes into the coherent system. While moving from source to drain electrons get scattered into the fictitious floating voltage probes and then re-emitted into the device by loosing the phase memory. To implement this idea each inelastic scatterer is modeled in terms of electron reservoir (or infinite impedance voltage probe) and coupled to the device. Since the net current flowing through the fictitious probes has to be zero to satisfy the conservation of total number of particles, electro-chemical potentials $(\mu)$ of each lead has to be adjusted accordingly. Due to its appealing simplicity Büttiker probe model has been extensively used in studying quantum transport in low-dimensional systems.

The idea of using molecules as active components of a device was suggested by Aviram and Ratner over three decades ago [14. Since then several ab-initio and model calculations have been performed to investigate molecular transport theoretically 15 23]. But experimental realizations took a little longer time to get feasible. In 1997, Reed and co-workers studied the current-voltage $(I-V)$ characteristics of a single benzene molecule attached to electrodes via thiol groups 24]. Various other experiments have been reported in literature exploring many interesting features e.g., ballistic transport, quantized conductance, negative differential resistance (NDR), gate controlled transistor operation to name a few. Effect of dephasing in molecular systems has been studied in few literatures both theoretically [25, 26] as well as experi- 
mentally. In 1989, Amato and Pastawski investigated the effect of inelastic scattering processes on a tight-binding linear disordered chain using Büttiker probe model [6]. In 2007, Datta et al. proposed a different phenomenological model which provides the flexibility of adjusting degree of phase and momentum relaxation independently [27]. In 2008, Nozaki et al. obtained transport properties through various molecular junctions by Büttiker model using Extended Hückel theory [28]. In 2009, Guo and co-workers have reported an ab-initio calculation 29] including the effect of phase breaking in 1,4-benzenedithiol molecule attached to two $\mathrm{Al}$ electrodes, gold QPC and a very thin $\mathrm{Al}(001)$ nanowire. They have also done a comparative analysis between Büttiker probe model and Datta dephasing model [27]. Though various efforts have already been made to explain the basic features of electron dephasing on molecular transport, but to the best of our knowledge, no rigorous effort has been made so far to unravel the combined effect of quantum interference and electron dephasing on molecular transport. This is the main motivation behind this work.

In our present article we study two terminal electron transport through a single benzene molecule including the effect of dephasing by a discrete tight-binding model. We obtain the transmission probability using Green's function technique within the framework of Landauer-Buttiker formulation. Transmittance-energy and current-voltage characteristics are obtained for three different configurations (para, ortho and meta) of benzene molecule depending on three different positions of the drain with respect to the source. The presence of dephasing provides a significant change in the spectral properties of the molecule and here we essentially focus our results in this aspect. Our model calculation can be extended further for any complicated molecular structure.

In what follows, we present the results. In section II, the model and the theoretical formulation are presented. Section III describes the results, and finally, we make our conclusions in section IV.

\section{MOLECULAR MODEL AND THEORETICAL FORMULATION}

We start by describing our model, illustrated in Fig. 1 where a single benzene molecule is connected symmetrically to two one-dimensional, semi-infinite leads, commonly known as source and drain. The leads are characterized by the electrochemical potentials $\mu_{S}$ and $\mu_{D}$, respectively, under the non-equilibrium condition when an external bias voltage is applied. Both the two leads and the molecule are simulated by a simple tight-binding Hamiltonian within nearest-neighbor hopping approximation. Following Büttiker's idea, the effect of dephasing due to inelastic scattering is incorporated in the model phenomenologically by coupling each spatial sites to a different fictitious electron reservoir or in other words an infinite impedance voltage probe (shown by the pink shaded area in Fig. 1) via perfect leads. The key idea is that the fictitious floating probes extract electrons from the device to the reservoir, but as the net current flowing through these probes is zero, so they re-inject the electrons into the conductor after phase randomization and thus effectively play the role of phase breaking scatterers.

The Hamiltonian for the entire system is given by the sum of four terms

$$
H=H_{\text {mol }}+H_{\text {leads }}+H_{\text {tun }}+H_{\text {dephase }} .
$$

The first term represents the Hamiltonian for the single benzene molecule consisting of six sites $(N=6)$ which is coupled to two electron reservoirs through conducting

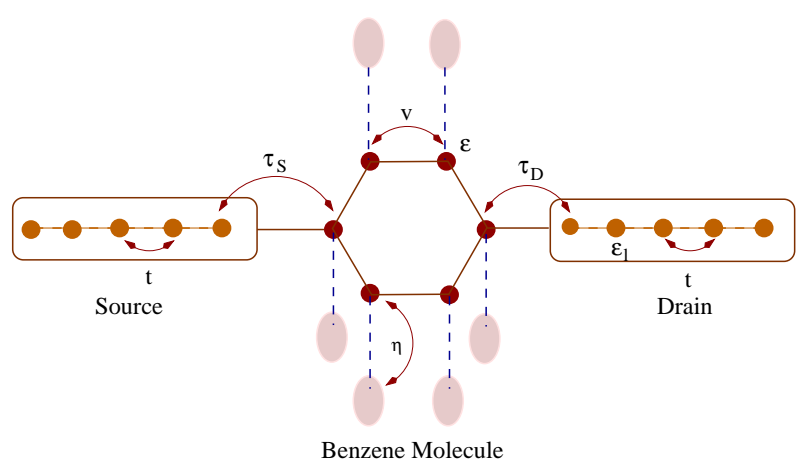

FIG. 1: (Color online). Schematic diagram of a single benzene molecule attached to two leads, namely, source and drain. Each molecular site is assumed to be coupled to a fictitious electron reservoir, shown by the pink shaded area, which takes into account the effect of phase breaking processes.

leads, namely, source and drain. The molecule is modeled by the nearest-neighbor tight-binding Hamiltonian which in Wannier basis can be written as,

$$
H_{m o l}=\sum_{i} \epsilon d_{i}^{\dagger} d_{i}+\sum_{i} v\left[d_{i+1}^{\dagger} d_{i}+\text { h.c. }\right]
$$

where $\epsilon$ refers to the site energy of an electron at the $i$-th site of the molecular system and $v$ represents the isotropic nearest-neighbor coupling strength between the molecular sites. $d_{i}^{\dagger}$ and $d_{i}$ correspond to the creation and annihilation operators, respectively, of an electron at the $i$-th site of the molecule.

Similarly the second and third terms of Eq. (1) denote the Hamiltonians for the one-dimensional semi-infinite leads (source and drain) and molecule-to-lead coupling. In Wannier basis representation they can be written as follows.

$$
\begin{aligned}
H_{\text {leads }} & =H_{S}+H_{D} \\
& =\sum_{\alpha=S, D}\left\{\sum_{n} \epsilon_{l} c_{n}^{\dagger} c_{n}+\sum_{n} t\left[c_{n+1}^{\dagger} c_{n}+\text { h.c. }\right]\right\}
\end{aligned}
$$


and,

$$
\begin{aligned}
H_{t u n} & =H_{S, m o l}+H_{D, \text { mol }} \\
& =\tau_{S}\left[d_{1}^{\dagger} c_{0}+\text { h.c. }\right]+\tau_{D}\left[d_{p}^{\dagger} c_{N+1}+\text { h.c. }\right] .
\end{aligned}
$$

Here, $\epsilon_{l}$ and $v$ stand for the site energy and nearestneighbor hopping between the sites of the two leads. $c_{n}^{\dagger}$ and $c_{n}$ are the creation and annihilation operators, respectively, of an electron at the site $n$ of the leads. The hopping integral between the source and the molecule is $\tau_{S}$, while it is $\tau_{D}$ between the molecule and the drain. Usually benzene molecules are attached to gold leads via thiol groups (-SH bond) in the chemisorption technique. Here, the effect of such substituent (e.g., thiol group) is included in the parameters $\tau_{S}$ and $\tau_{D}$. The source is assumed to be connected at the site number 1 , while the drain is coupled to the $p$-th site, which is variable, of the molecular system. The integer $p$ becomes 2,3 and 4 for the ortho, meta and para configurations, respectively.

In order to incorporate the effect of dephasing, external electron reservoirs are phenomenologically introduced and they are connected to all the molecular sites (six) through fictitious probes. The phase breaking probes are identically represented within tight-binding framework like source and drain as given below.

$$
H_{\text {dephase }}=\sum_{i=1}^{N}\left[H_{i}^{d, \text { probes }}+H_{i}^{d, t u n}\right],
$$

where,

$$
H_{i}^{d, \text { probes }}=\sum_{m} \epsilon_{d} c_{m}^{D i \dagger} c_{m}^{D i}+\sum_{m} t_{d}\left[c_{m+1}^{D i \dagger} c_{m}^{D i}+h . c .\right]
$$

and,

$$
H_{i}^{d, t u n}=\eta\left[c_{0}^{D i \dagger} d_{i}+\text { h.c. }\right] .
$$

Here, $\epsilon_{d}$ and $t_{d}$ being the site energy and the hopping strength between the nearest-neighbor sites of the dephasing probes, respectively. $c_{m}^{D i \dagger}\left(c_{m}^{D i}\right)$ is the creation (annihilation) operator of an electron at the $m$-th site of the $i$-th lead. The dephasing strength is characterized by the coupling parameter $\eta$.

To obtain the transmission probability of an electron through such a molecular bridge system, we use Green's function formalism. Within the regime of coherent transport and in the absence of Coulomb interaction this technique is well applied.

The single particle Green's function operator representing the entire system for an electron with energy $E$ is defined as,

$$
G=(E-H+i \eta)^{-1}
$$

where, $\eta \rightarrow 0^{+}$.

Following the matrix form of $\boldsymbol{H}$ and $\boldsymbol{G}$ the problem of finding $\boldsymbol{G}$ in the full Hilbert space $\boldsymbol{H}$ can be mapped exactly to a Green's function $G_{\text {mol }}^{\text {eff }}$ corresponding to an effective Hamiltonian in the reduced Hilbert space of the molecule itself and we have,

$$
\mathcal{G}=G_{m o l}^{\text {eff }}=\left(E-H_{m o l}-\Sigma_{S}-\Sigma_{D}-\sum_{k=1}^{N} \Sigma_{k}\right)^{-1}
$$

where,

$$
\begin{aligned}
\Sigma_{S(D)} & =H_{S, \operatorname{mol}(\operatorname{mol}, R)}^{\dagger} G_{S(D)} H_{S, m o l(m o l, R)}, \\
\Sigma_{k} & =H_{k}^{d, t u n \dagger} G_{k} H_{k}^{d, t u n} .
\end{aligned}
$$

These $\boldsymbol{\Sigma}_{\boldsymbol{S}(\boldsymbol{D})}$ and $\boldsymbol{\Sigma}_{\boldsymbol{k}}$ are the contact self-energies introduced to incorporate the effect of coupling of the molecule to the source (drain) and the fictitious electron reservoirs. It is evident from Eq. (10) that the form of the selfenergies are independent of the molecule itself through which transmission is studied. Using Dyson equation the analytic form of the self energies can be evaluated as follows,

$$
\Sigma_{\nu}=\frac{\tau_{\nu}^{2}}{E-\epsilon_{\nu}-\sigma_{\nu}} .
$$

Here, $\sigma_{\nu}=\left(E-\epsilon_{\nu}\right) / 2-i \sqrt{t_{\nu}^{2}-\left(E-\epsilon_{\nu}\right)^{2} / 4}$; and $\nu=$ $S, D, 1,2, \ldots N$. For source and drain, $\nu=S(D), \tau_{\nu}=$ $\tau_{S(D)}, \epsilon_{\nu}=\epsilon$, and $t_{\nu}=t$; while considering the dephasing probes, $\nu=k(k=1,2, \ldots N), \tau_{\nu}=\eta, \epsilon_{\nu}=\epsilon_{l}$, and $t_{\nu}=t_{d}$.

Following D'Amato-Pastawski model [ [6] the effective transmission probability of an electron from source to drain including the partially phase breaking process is given by the following expression.

$$
T_{e f f}=T_{S, D}+\sum_{i, j=1}^{N} \boldsymbol{T}_{D, i} \boldsymbol{W}_{i, j}^{-1} \boldsymbol{T}_{j, S} .
$$

The above expression has a significant physical implication. The first term is the coherent contribution to electron transmission, whereas the second term refers to the incoherent component of tunneling due to electrons suffering phase randomizing scattering at the fictitious dephasing reservoirs. $T_{i, j}$ is the transmission probability between any pair of reservoirs $i, j(i, j=S, D, 1,2 \ldots N)$ and it is expressed as follows,

$$
T_{i, j}=\operatorname{Tr}\left[\boldsymbol{\Gamma}_{\boldsymbol{i}} \mathcal{G}^{r} \boldsymbol{\Gamma}_{\boldsymbol{j}} \mathcal{G}^{\boldsymbol{a}}\right] .
$$

$\Gamma_{i}$ 's are the coupling matrices representing the coupling between the molecule and the leads and they are mathematically defined by the relation,

$$
\Gamma_{i}=i\left[\Sigma_{i}^{r}-\Sigma_{i}^{a}\right]
$$

Here, $\boldsymbol{\Sigma}_{\boldsymbol{k}}^{r}$ and $\boldsymbol{\Sigma}_{\boldsymbol{k}}^{a}$ are the retarded and advanced selfenergies associated with the $k$-th lead, respectively.

In the second term of Eq. (12), $W$ matrix is defined by the following relation,

$$
W_{i, j}=\left[\left(1-R_{i, i}\right) \delta_{i j}-T_{i, j}\left(1-\delta_{i j}\right)\right],
$$


where,

$$
R_{i, i}=1-\sum_{j \neq i} T_{i, j}
$$

$R_{i, i}$ is the reflection probability of an electron from $i$-th lead.

It is shown in literature by Datta et al. [3, 4] that the self-energy can be expressed as a linear combination of a real and an imaginary part in the form,

$$
\boldsymbol{\Sigma}_{j}^{r}=\boldsymbol{\Lambda}_{j}-i \boldsymbol{\Delta}_{j}
$$

The real part of self-energy describes the shift of the energy levels and the imaginary part corresponds to the broadening of the levels. The finite imaginary part appears due to incorporation of the semi-infinite leads having continuous energy spectrum. Therefore, the coupling matrices can easily be obtained from the self-energy expression and is expressed as,

$$
\boldsymbol{\Gamma}_{\boldsymbol{i}}=-2 \operatorname{Im}\left(\boldsymbol{\Sigma}_{\boldsymbol{i}}\right)
$$

Considering linear transport regime, at absolute zero temperature the linear conductance $\left(g_{e f f}\right)$ is obtained using two-terminal Landauer conductance formula,

$$
g_{e f f}=\frac{2 e^{2}}{h} T_{e f f}\left(E_{F}\right) .
$$

With the knowledge of the effective transmission probability we compute the current-voltage $(I-V)$ characteristics by the standard formalism based on quantum scattering theory.

$$
I(V)=\frac{2 e}{h} \int_{-\infty}^{\infty} T_{e f f}\left[f_{S}(E)-f_{D}(E)\right] d E .
$$

Here, $f_{S(D)}(E)=\left[1+e^{\frac{E-\mu_{S(D)}}{k_{B} T}}\right]^{-1}$ is the Fermi function corresponding to the source and drain. At absolute zero temperature the above equation boils down to the following expression.

$$
I(V)=\frac{2 e}{h} \int_{E_{F}-\frac{e V}{2}}^{E_{F}+\frac{e V}{2}} T_{e f f} d E .
$$

In our present work we use the above expression assuming that the potential drop takes place only at the boundary of the molecular system. This assumption is good enough for molecules of smaller size. Throughout our study we choose $c=e=h=1$ for the sake of simplicity.

\section{NUMERICAL RESULTS AND DISCUSSION}

In this section we present the results obtained by numerical simulation considering a single benzene molecule sandwiched between source and drain. The orbital energies $(\epsilon)$ of the molecule are set to zero and the nearestneighbor hopping matrix element $(t)$ is fixed at $2.4 \mathrm{eV}$ [17] for an aromatic ring structure of the benzene molecule. The other hopping parameters in the leads $(t)$ and the dephasing probes $\left(t_{d}\right)$ are fixed at $4 \mathrm{eV}$, while the site energies are chosen to be zero i.e., $\epsilon_{l}=\epsilon_{d}=0$. The dephasing strength, characterized by the parameter $\eta$, is fixed at $1 \mathrm{eV}$. Throughout our analysis the coupling strengths

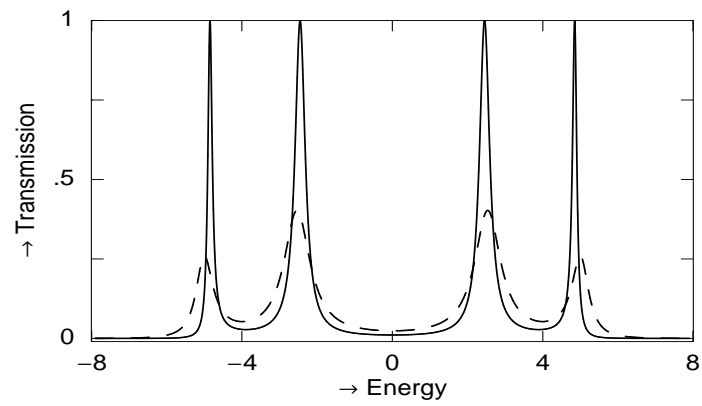

FIG. 2: Variation of transmission probability as a function of energy for a benzene molecule where the drain is connected at the para position with respect to the source. The solid and dashed curves correspond to the results in the absence and presence of dephasing, respectively.

of the molecule to the source and drain, characterized by the parameters $\tau_{S}$ and $\tau_{D}$, are set at $1 \mathrm{eV}$. The molecular coupling to the contacting leads is one of the most important factors which regulates the electronic transmission through a molecular wire and this phenomenon has been extensively studied in our previous works [22, 23].

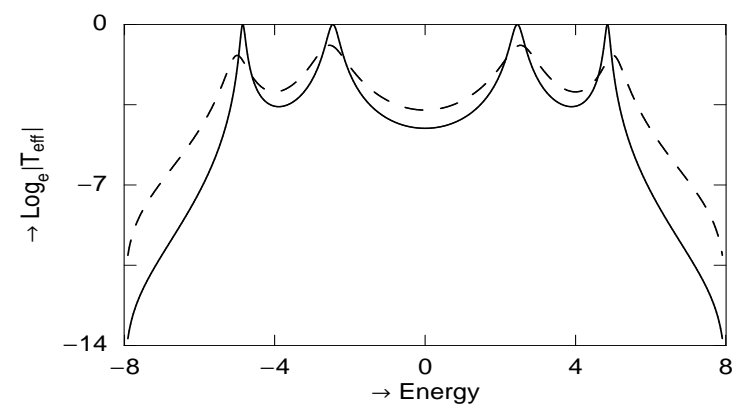

FIG. 3: The results of Fig. 2 are re-plotted in a logarithmic scale to clarify the effect of dephasing more clearly.

Accordingly, here we describe all the essential features of electron transport for a particular molecular coupling strength. The molecular transport properties are also highly sensitive on the molecule-to-lead interface geometry, and, in the present work we illustrate how they are influenced in the presence of electron dephasing. To reveal these facts first we discuss the behavior of transmittanceenergy characteristics and then we describe the nature of current-voltage spectra for three different configurations (ortho, meta and para) of the benzene molecule.

Here, we establish all the results at absolute zero tem- 
perature and restrict ourselves within elastic dephasing so that no energy is exchanged between the transported electrons and the external fictitious reservoirs, only the phase informations get lost. This condition allows the incoherent electrons to generate a steady-state current through the sample.

\section{A. Transmittance-energy characteristics}

\section{Molecule coupled symmetrically}

In Fig. 2 we show the variation of transmission probability as a function of injecting electron energy for a benzene molecule, where the molecule is coupled symmetrically to the source and drain i.e., the upper and

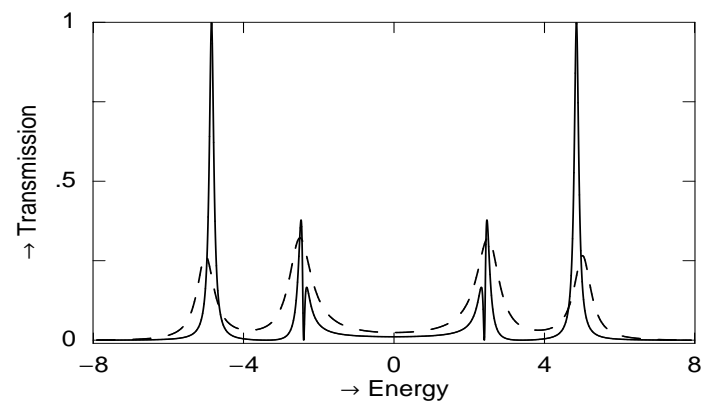

FIG. 4: Transmission probability versus energy characteristics of a benzene molecule where the drain is connected at the ortho position with respect to the source. The solid and dashed lines represent the results in the absence and presence of dephasing, respectively.

lower arms of the molecular ring have identical length. This is the so-called para configuration. The solid curve represents the variation of coherent transmission proba-

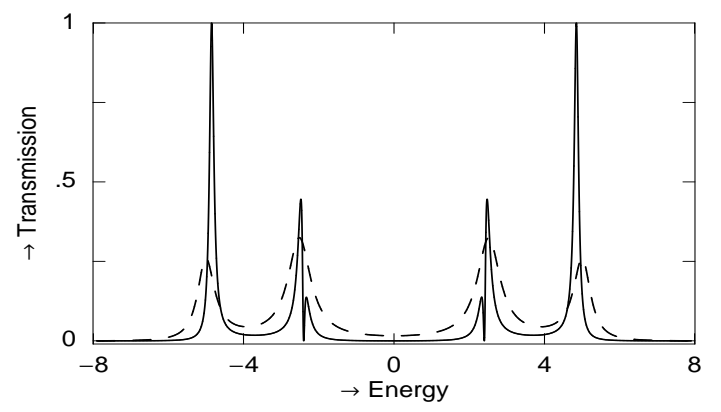

FIG. 5: Transmission probability versus energy characteristics of a benzene molecule where the drain is coupled to the meta position with respect to the source. The solid and dashed curves correspond to the results in the absence and presence of dephasing, respectively.

bility, while the dashed curve depicts the result in the presence of electron dephasing. It is observed that, in the absence of dephasing the transmission probability exhibits sharp resonant peaks (see solid line) for some spe- cific energies, whereas it almost vanishes for all other energy values. At these resonances, the transmission probability $T_{\text {eff }}$ goes to unity. All the resonant peaks are associated with the energy eigenvalues of the benzene molecule, and therefore, we can say the transmittance spectrum is a fingerprint of the electronic structure of the molecule. The situation becomes much interesting when the effect of dephasing is introduced. It shows that the magnitude of the resonant peaks gets suppressed enor-

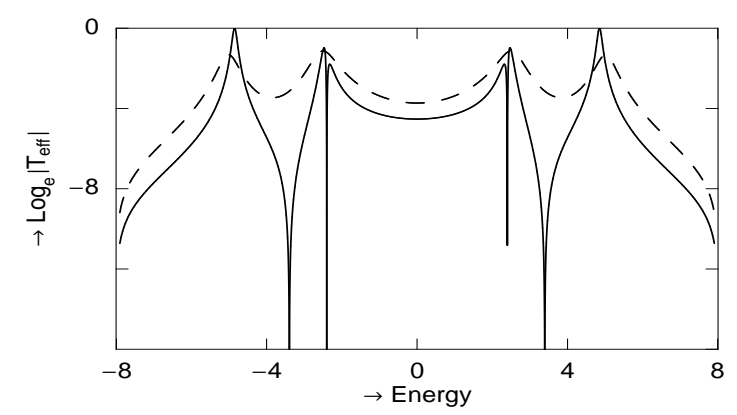

FIG. 6: The results of Fig. 4 are re-plotted in a 'log' scale.

mously (dashed line) compared to the coherent transmission (solid line). This is due to the increased rate of scattering in the additional fictitious electron reser-

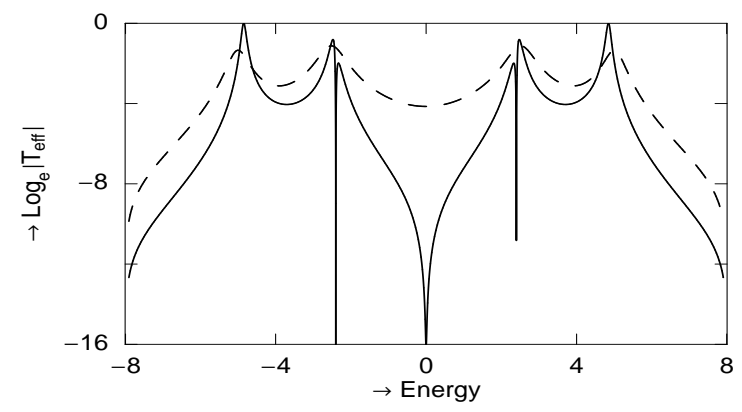

FIG. 7: The results of Fig. [5 are re-plotted in a 'log' scale.

voirs. Additionally, we also notice that the widths of the resonant peaks become broadened. This broadening is caused by the dominance of phase randomizing effect over backscattering due to loss of phase coherence of interfering electrons in the presence of dephasing reservoirs. To judge the effect of dephasing more transparently, in Fig. 3 we re-plot the results of Fig. 2 in a logarithmic scale, where the solid and dashed lines represent the identical meaning as above. Since the transmission probability lies within the range 0 to 1 , it becomes zero or negative in the logarithmic scale as shown in Fig. 3 It is observed that the variations in the transmission peaks between the two curves (solid and dashed) get suppressed, but the broadening effect in the presence of dephasing can be much clearly observed from the 'log' scale. 


\section{Molecule coupled asymmetrically}

A significant change in the transmission spectrum is observed when the benzene molecule is coupled asymmetrically to the source and drain i.e., the upper and lower arms of the molecular ring have unequal lengths. For the ortho configuration the results are given in Fig. 4. while in Fig. 5 the results are shown for the meta configuration. From the spectra (Figs. 4 and 5) we notice that in the absence of dephasing some resonant peaks do not reach to unity and their amplitudes are significantly reduced compared to the other resonant peaks. This is solely due to the effect of quantum interference between the electronic waves passing through the upper and lower arms of the molecular ring. Quite interestingly we observe that in these asymmetric molecular wires (ortho and meta) two anti-resonant states appear in the transmittance spectrum where transmission probability drops exactly to zero. These anti-resonant states are specific to the interferometric nature of the molecular sys-

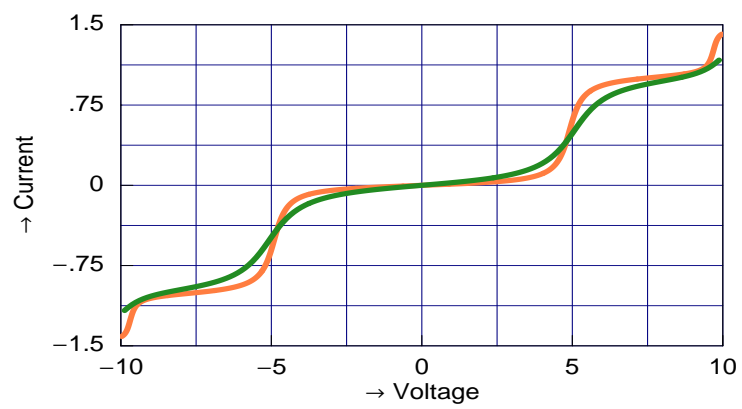

FIG. 8: (Color online). $\quad I-V$ characteristics of a benzene molecule where the drain is connected at the para position with respect to the source. The orange and green curves correspond to the currents in the absence and presence of dephasing, respectively.

tem and their positions in the energy scale are independent of the molecule-to-lead coupling strength. A careful observation reveals that a sharp peak is followed by a sharp dip and vice versa across the anti-resonant energy which is quite analogous to a Fano-like line shape. All these anti-resonant states disappear as long as dephasing of electrons is taken into account those are clearly observed from the dashed lines of Figs. 4 and 5 . Here also the magnitudes of the resonant peaks get decreased and they become broadened as we describe earlier in the para configuration. The results of Figs. 4 and 5 are redrawn in Figs. 6 and 7 respectively, where the transmission probabilities are determined in the 'log' scale. From these spectra (Figs. 6]and 7) the characteristic features of anti-resonant states in the case of coherent transmission and the broadening of resonant peaks in the presence of electron dephasing are clearly noticed. Our results also predict that in the asymmetric molecular ring the positions of the anti-resonant states are strongly sensitive on the location of measuring electrodes. Broadening of transmission peaks in presence of dephasing is much pronounced in the case where the drain is connected at the meta position of the benzene molecule rather than the ortho position, and, it can be clearly observed from the $\log _{e} T_{\text {eff }}$ vs. E plot instead of the transmittance-energy spectra.

\section{B. Current-voltage characteristics}

All the above features of electron transmission become much clear from our study of current-voltage $(I-V)$ characteristics. The current through the molecular system is obtained by integrating the transmission function following Eq. (21). Here we fix the Fermi energy $E_{F}$ at zero.

\section{Molecule coupled symmetrically}

In Fig. 8 we plot the variation of current as a function of applied bias voltage for a benzene molecule where the leads are connected symmetrically. The orange and green lines represent the currents in the absence and presence of electron dephasing. The current varies quite continuously with the applied bias voltage $V$. Depending

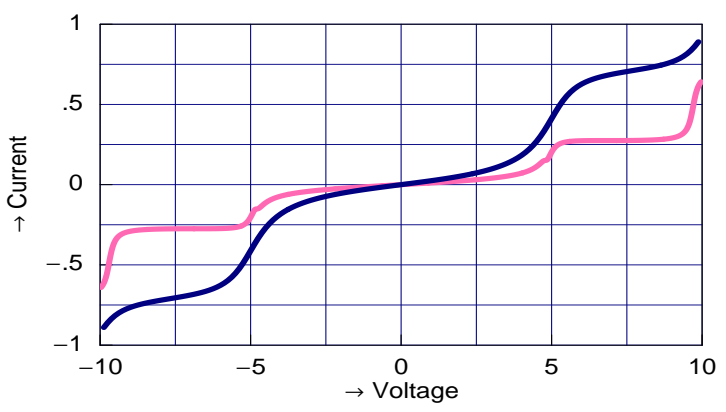

FIG. 9: (Color online). $I-V$ characteristics of a benzene molecule where the drain is connected at the ortho position with respect to the source. The pink and deep blue lines represent the currents in the absence and presence of dephasing, respectively.

on the molecular coupling to the side attached leads, the current shows continuous-like or step-like behavior [22]. Tuning the molecular coupling strength the current amplitude through the molecular wire can be regulated nicely for a fixed bias voltage. This provides an interesting behavior in designing molecular electronic devices. Both in the absence and presence of electron dephasing current shows almost identical variation though a significant change is observed in their conductance spectra. This is due to the fact that the dephasing broadens the transmission peaks, while it also suppresses the magnitude. These two effects nullify each other showing almost identical spectrum in the $I-V$ characteristics. 


\section{Molecule coupled asymmetrically}

The effect of dephasing becomes much prominent in the current-voltage characteristics when the molecule is coupled asymmetrically to the source and drain. For the ortho configuration, the currents are shown in Fig. 9 , while in Fig. 10 the results are shown when the molecule is coupled to the leads in the meta configuration. Quite similar to the para configuration (Fig. 8), in these two cases (ortho and meta configurations) also the current varies almost continuously with the applied bias voltage $V$. In these asymmetric configurations, the current amplitude gets enhanced significantly when the phase breaking effect is considered (deep blue curve in Fig. 9 and

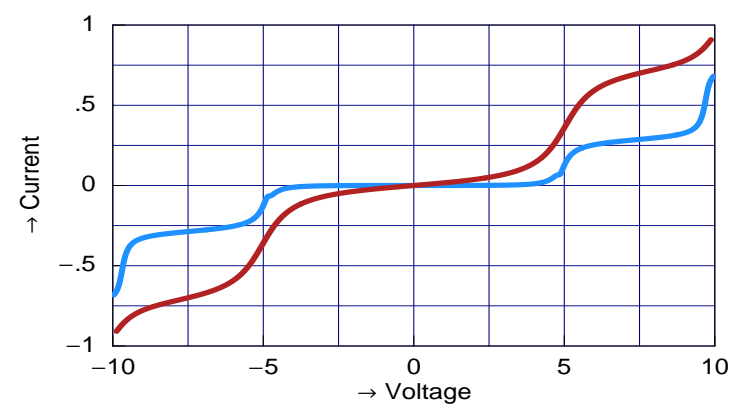

FIG. 10: (Color online). $I-V$ characteristics of a benzene molecule where the drain is connected at the meta position with respect to the source. The light blue and red lines correspond to the currents in the absence and presence of dephasing, respectively.

light blue curve in Fig. 10 compared to the case where the molecule is free from any phase randomizing process (pink line in Fig. 9 and red line in Fig. 10). Such an enhancement in current amplitude takes place because of notable broadening of the transmission peaks due to reduced destructive interference for dominance of phase decoherence over the backscattering effect.

\section{CLOSING REMARKS}

To summarize, in the present work we have examined electron transport phenomena through a single benzene molecule including the effect of phase breaking using a phenomenological model introduced by Büttiker. Experimentally the benzene molecule is usually coupled to noble metal electrodes (e.g., gold leads) through thiol linking groups. In our theoretical work, we present the entire system (source-molecule-drain) by a discrete lattice model within a simple one-electron, tight-binding framework. We have studied transmission-energy spectrum and current-voltage characteristics considering three different configurations of the system depending on the position of the drain contact (ortho, meta and para positions). We have observed that the sharp transmission peaks are broadened and their magnitude is also sup- pressed in the presence of dephasing due to loss of phase coherence and thus it provides an enhancement in the current amplitude. But the broadening effect and so as the increase in current amplitude is much pronounced in the case where the drain is connected asymmetrically to the molecule with respect to the source. Another significant feature is that the two anti-resonant states, observed in the transmittance spectrum of an asymmetrically connected benzene molecule, completely disappear in the presence of strong dephasing due to reduced destructive interference. Thus the effect of dephasing can provide a significant change in the current-voltage relation.

In the Büttiker probe model fictitious voltage probes are introduced into the coherent system. These virtual probes introduce additional resistance in the system by relaxing the momentum of the electrons by partially reflecting them i.e., back-scattering. This model is appropriate for those molecular systems where the dominant scattering source does not conserve electron momentum, for example, electron-phonon scattering which is very important in molecular conductor in finite temperature. However, Büttiker model does not consider the effect of momentum relaxation. In experimental situations dephasing may also arise from electron-electron scattering which destroy phase but not momentum [27, 29]. Datta model presents the flexibility of tuning phase and momentum independently of each other and we intend to study this phenomenological model in near future.

Recent experimental progresses have significantly inspired in model calculations and numerical simulations to study vibronic effects on electron transport in molecular junctions 30 33]. Most of the studies of vibronic effects in the resonant tunneling regime (for higher voltages) have illustrated that vibrational motion may affect the current-voltage spectra quite significantly. But, the point is that in the resonant tunneling region vibronic effect becomes significant only when the molecule is coupled to the measuring leads weakly. In a very recent work [34] Benesch et al. have studied vibronic effects on conductance in molecular junctions where a benzene ring is coupled weakly to two gold electrodes and shown that the vibronic motion affects current-voltage spectra. On the other hand, when the molecule is coupled strongly to the electrodes, the vibrational effects in resonant transport are almost negligible. This phenomenon has been clearly justified in the reference 35]. In the present work we have investigated all the essential features of electron transport in the limit of strong molecule-to-electrode coupling, and accordingly, we neglect the vibronic effect.

Throughout our work we have neglected the intra- and inter-site Coulomb interactions as well as the effect of the leads, which we wish to consider in our future studies. Another important assumption is the zero temperature approximation. Although the electron-phonon interaction becomes significant at non-zero temperature, but in our case, we have already considered the dephasing effect phenomenologically at every molecular sites, 
which takes into account the effect of dephasing of electrons without any exchange of energy between the electron and the external leads. Accordingly, we compute the current-voltage relation at absolute zero temperature. At finite non-zero temperature, the transmission peaks will broaden due to thermal broadening effect, but the basic features will not change significantly as long as the thermal energy $\left(k_{B} T\right)$ is less than the average level spacing of the benzene molecule. Now-a-days various organic compounds and polymers (for example,
Tris(8-hydroxyquinolino) aluminum ( $\left.\mathrm{Alq}_{3}\right)$ and 1,2,3,4,5pentaphenylcyclopentadiene (PPCP)) are used as electroluminescent devices. Knowledge of electronic structure and current-voltage relation is important to elucidate the light emitting mechanism [36]. It may be interesting to see the effect of electron dephasing on this mechanism. Our presented results may be useful in understanding the effect of phase breaking processes on the basic two-terminal molecular transport phenomena.
[1] A. Nitzan and M. A. Ratner, Science 300, 1384 (2003).

[2] F. Chen and N. J. Tao, Acc. Chem. Res. 42, 429 (2009).

[3] S. Datta, Electronic transport in mesoscopic systems, Cambridge University Press, Cambridge (1995).

[4] S. Datta, Quantum Transport: Atom to Transistor, Cambridge University Press, Cambridge (2005).

[5] R. Landauer, IBM J. Res. Dev. 1, 223 (1957).

[6] J. L. D'Amato and H. M. Pastawski, Phys. Rev. B 41, 7411 (1990).

[7] B. C. Stipe, M. A. Rezaei, and W. Ho, Science 280, 1732 (1998).

[8] W. Wang, T. Lee, I. Kretzschmar, and M. A. Reed, Nano Lett. 4, 643 (2004).

[9] M. Galperin, M. A. Ratner, and A. Nitzan, J. Phys.: Condens. Matter 19, 103201 (2007).

[10] N. Sergueev, D. Roubtsov, and H. Guo, Phys. Rev. Lett. 95, 146803 (2005).

[11] N. Sergueev, A. A. Demkov, and H. Guo, Phys. Rev. B. 75, 233418 (2007).

[12] M. Büttiker, Phys. Rev. B 33, 3020 (1986).

[13] M. Büttiker, IBM J. Res. Dev. 32, 63 (1988).

[14] A. Aviram and M. Ratner, Chem. Phys. Lett. 29, 277 (1974).

[15] M. D. Ventra, S. T. Pentelides, and N. D. Lang, Appl. Phys. Lett. 76, 3448 (2000).

[16] M. D. Ventra, N. D. Lang, and S. T. Pentelides, Chem. Phys. 281, 189 (2002).

[17] D. M. Cardamone, C. A. Stafford, and S. Mazumdar, Nano Lett. 6, 2422 (2006).

[18] K. Tagami, L. Wang, and M. Tsukada, Nano Lett. 4, 209 (2004).

[19] P. Orellana and F. Claro, Phys. Rev. Lett. 90, 178302 (2003).

[20] J. H. Ojeda, R. P. A. Lima, F. Domnguez-Adame, and
P. A. Orellana, J. Phys.: Condens. Matter 21, 285105 (2009).

[21] M. Araidai and M. Tsukada, Phys. Rev. B 81, 235114 (2010).

[22] S. K. Maiti, Phys. Lett. A 366, 114 (2007).

[23] P. Dutta, S. K. Maiti, and S. N. Karmakar, Org. Electron. 11, 1120 (2010).

[24] M. A. Reed, C. Zhou, C. J. Muller, T. P. Burgin, and J. M. Tour, Science 278, 252 (1997).

[25] X.-Q. Li and Y. J. Yan, Appl. Phys. Lett. 79, 2190 (2001).

[26] X.-Q. Li and Y. J. Yan, J. Chem. Phys. 115, 4169 (2001).

[27] R. Golizadeh-Mojarad and S. Datta, Phys. Rev. B 75, 081301(R) (2007).

[28] D. Nozaki, Y. Girard, and K. Yoshizawa, J. Phys. Chem. C 112, 17408 (2008).

[29] J. Maassen, F. Zahid, and H. Guo, Phys. Rev. B 80, 125423 (2009).

[30] A. Pecchia and A. D. Carlo, Nano Lett. 4, 2109 (2004).

[31] Y. Chen, M. Zwolak, and M. D. Ventra, Nano Lett. 5, $813(2005)$.

[32] J. Bonca and S. A. Trugman, Phys. Rev. Lett. 75, 2566 (1995).

[33] J. Koch and F. von Oppen, Phys. Rev. Lett. 94, 206804 (2005).

[34] C. Benesch, M. Cizek, J. Klimes, I. Kondov, M. Thoss, and W. Domcke, J. Phys. Chem. C 112, 9880 (2008).

[35] C. Benesch, M. Cizek, M. Thoss, and W. Domcke, Chem. Phys. Lett. 430, 355 (2006).

[36] K. Sugiyama, D. Yoshimura, T. Miyamae, T. Miyazaki, H. Ishii, Y. Ouchi, and K. Seki, J. Appl. Phys. 83, 4928 (1998). 